\title{
Biochemical risk markers for ischemic heart diseases in diabetic patients
}

\author{
Ihsan Hassan Al-Dabbagh, Raad Yahya Al-Hamdani
}

Department of Biochemistry. College of Medicine, University of Mosul

Received: $\mid v / V / r \ldots o$; Accepted: $r / 1 . / r \ldots 0$

\begin{abstract}
Fasting blood samples were collected from $1 \leqslant r$ diabetics ( ${ }^{\vee} \vee$ males, 10 females) included $1 \cdot(\vee \%)$ with type 1 and $I r r(q \% \%)$ with type $r$ diabetes, age ranged $1 \cdot-\wedge$. with mean \pm SD $01 . V_{ \pm}$I $Y$. $r$ years. They were attending AL-Waffa diabetic clinic in Mosul during the period from ${ }^{1 \text { st }}$ November $r_{\ldots} . r_{-}{ }^{\text {st }}$ April $r \ldots \varepsilon$. A control group of 110 apparently healthy non-diabetic volunteers ( 77 females, $\leqslant 9$ males ), age ranged $1 \cdot$. $\checkmark \cdot$ with mean \pm SD $r 0.0_{ \pm} \mid Y . V$ years were included for comparison.
\end{abstract}

A statistical significant differences $(\mathrm{p}<\bullet .0)$ were detected in fasting plasma glucose,glycated hemoglobin, serum totalcholesterol, triglycerides, low density lipoprotein cholesterol and high density lipoprotein cholesterol between diabetics and the control group. The diabetic group had a mixed hyperlipidemia. They were more obese and had higher blood pressure than control group. Hypertensive diabetics seemed to have the highest risk within the diabetics themselves as they had their total cholesterol and low density lipoprotein cholesterol significantly higher than non hypertensive diabetics. Diabetic patients with ischemic heart disease generally had longer duration of Diabetes Mellitus than those diabetics with out ischemic heart disease . Tight control of blood pressure together with optimal glycemic control is mandatory in this group.

الخلص مة

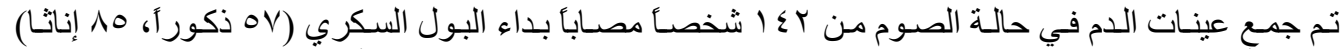

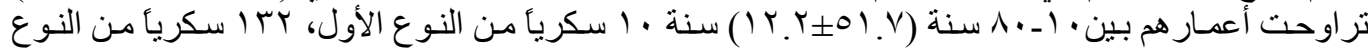

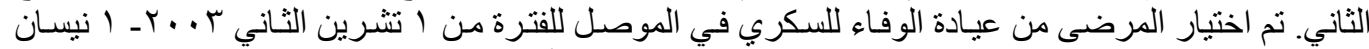

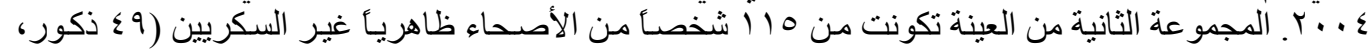

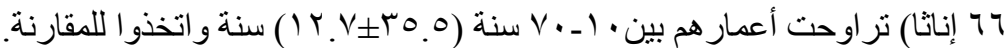

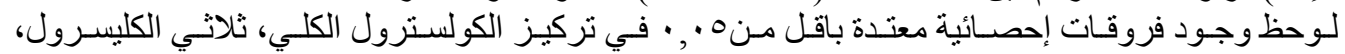

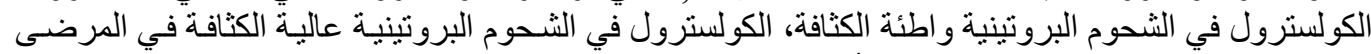

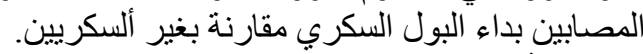

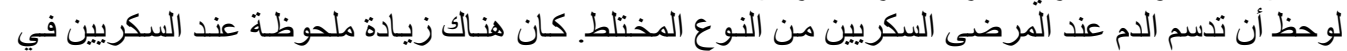

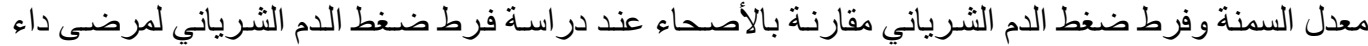

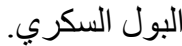

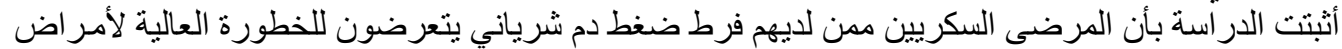

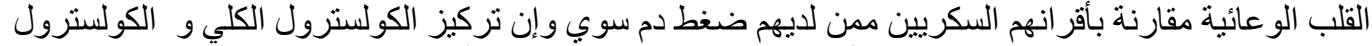

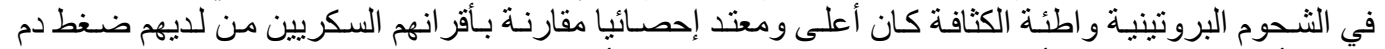

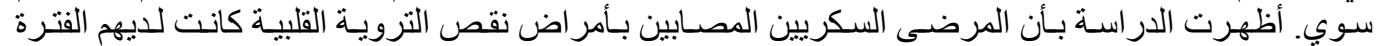

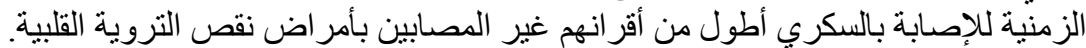

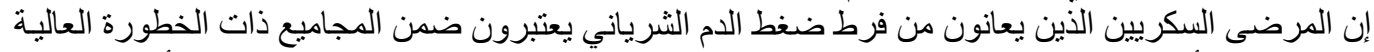

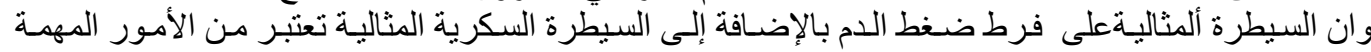
والضرورية. 


\section{Introduction}

D iabetes mellitus (DM) is a clinical syndrome characterized by hyperglycemia due to absolute or relative deficiency of insulin ('). It is not only the deficiency of insulin but along with varying degrees of peripheral resistance to its action ${ }^{(\Upsilon)}$.

Death may result from acute metabolic decompansation while long standing metabolic derangement is frequently associated with permanent and irreversible functional and structural damage in the cells of the body, with those of the vascular system, being particularly susceptible ${ }^{(1)}$.

Adults with diabetes have an annual mortality rate of $0 . £ \%$ - twice that of those without the condition- and their life expectancy is decreased by five to ten years. This increased mortality is due to atherosclerotic vascular complication $^{(\tau)}$.

Ischemic heart disease(IHD) is caused by the same risk factors found in general population but these risks become magnified by high blood glucose. High blood glucose creates harmful changes in low density lipoprotein (LDL), high density lipoprotein (HDL), triglycerides, increases clotting, elevates blood pressure and alters blood flow. People with diabetes have other as yet unexplained risk, the standard heart risk magnified by high blood glucose, do not explain all of the excess heart damage seen in diabetics ${ }^{(\xi)}$.

Although the treatment of diabetes has traditionally focused on glycemic control for reducing microvascular complication, recent attention has also focused on reducing risks of macro vascular complications (namely IHD and stroke) ${ }^{\left({ }^{\circ}\right.}$. The prevention of IHD is based on the control of several factors associated with a disease and suspected to play a pathogenetic role, defined as "risk factors" often quoted risk factors include obesity, insulin resistance, smoking, abnormality of lipid metabolism and hypertension ${ }^{(7)}$. The available interventions to reduce IHD and stroke incidence among diabetics include aggressive blood pressure control and reduction in serum cholesterol $^{(\circ)}$.

The aim of the present study is to determine biochemical risk markers for ischemic heart diseases among diabetics and non diabetics , and to assess the effect of hypertension on these markers among diabetics and non diabetics.

\section{Subjects \&Methods}

Subjects: This study was conducted in Mosul during the period from the ${ }^{1 \text { st }}$ of November $r \ldots r$ to the $1^{\text {st }}$ of April r.. . The study was divided into two groups:

1- Non diabetic group (group'): one hundred fifteen apparently healthy subjects, included forty nine males, sixty six females, age ranged between $1 \cdot-v \cdot$ years . Confirmation for absence of DM was done by measuring their fasting plasma glucose. They were matched for age, family history for diabetes and for familial hyperlipidemia.

r- Diabetic group (Group II): one hundred forty two diabetic patients consisted of fifty seven males and eighty five females, age ranged between 1.-A. years.

Individuals of this group were diagnosed previously to have DM and they were regular attendants of AlWaffa diabetic clinic in Mosul for checking of their disease control and treatment. The subjects of both groups were interviewed and the data collected included name, age, occupation, residence, family history, type of diabetes, duration of the disease, type of treatment, drug history, medical history and smoking habbits. The following are the details of collected data:

'.Blood pressure measurement: Hypertension was diagnosed on the base of history and treatment if patient was hypertensive in the past, and for new cases, we labeled them hypertensive if blood pressure is equal to or more than 1 $\varepsilon \cdot / 9 \cdot \mathrm{mmHg}^{(v)}$. Mode of treatment of 
hypertension was not included as a criterion of classification.

$r$. Body mass index: body mass index BMI was calculated according to the formula. BMI = weight $(\mathrm{kg}) /$ height $\left(\mathrm{m}^{r}\right)^{(\wedge)}$

$r$. Presence of IHD: History of IHD was taken from subjects in both groups and they were considered to have IHD if previously diagnosed by physician and they were on treatment of IHD.

Methods: Fasting blood samples were taken as $\wedge \mathrm{ml}$ venous blood of an overnight fasting state. The blood samples were divided into three parts, each of which was treated in different ways as follows:

For glucose measurement $1 \mathrm{ml}$ was transferred into fluoride tube containing sodium fluoride to inhibit glycolsis, potassium oxalate as anticoagulant. For $\mathrm{HbA}$ ' c measurement, ' $\mathrm{ml}$ of blood was transferred into EDTA tube, with gentle shaking for proper mixing with EDTA, to obtain whole blood sample that was used for glycated hemoglobin measurement and the remaining $7 \mathrm{ml}$ of blood transferred into disposable plain tube, allowed to clot for $10_{-} r \cdot$ minutes in water bath at $r v^{\circ} \mathrm{C}$, then serum was separated by centrifugation for the measurement of other biochemical parameters (Sera were frozen at $-r \cdot{ }^{\circ} \mathrm{C}$ and kept for analysis at weekly patches)Plasma glucose was measured by oxidase peroxides $\operatorname{method}^{\left({ }^{9}\right)}$ Glycated hemoglobin measured in whole blood sample by ion exchange resin quantitative colorimetric determination ${ }^{(\cdot)}$, using a kit supplied from stanbio (USA).Uric acid was determined enzymaticaly by spectrophotometric $\operatorname{method}^{(1)}$ using a kit purchased from biomerieux (France).

Total cholesterol and triglycerides were measured by enzymatic method ${ }^{(\uparrow)}$ sing kits purchased from Biomerieux (France)The chylomicrons and lipoproteins of VLDL and LDL contained in the sample are precipitated by the addition of phosphotungstic acid in the presence of magnesium ions. The supernatant obtained after centrifugation contains HDL from which the cholesterol can be measured using cholesterol esterase and oxidase (as in total cholesterol measurement) ${ }^{(1 r)}$.

LDL-C can be determined from the difference between total cholesterol, VLDL and HDL cholesterol present in the supernatant after the precipitation of LDL fraction with heparin at their isoelectric point $\mathrm{PH} 0^{\circ} \cdot \varepsilon^{(1 \xi)}$. The statistical methods used were, Paired student z-test, unpaired student $\mathrm{Z}$ test $^{\left({ }^{\circ}\right)}$. Multiple logistic analysis was used to examine the association between the parameters of IHD and any predicator believed to influence it and Chi-squared test using $r_{X}{ }^{r}$ contiguency table was used to compare any two $\operatorname{groups}^{(17)}$.

All values quoted as the mean \pm SD . Differences between observation were considered significant at $\mathrm{P}<\cdot . \bullet$.

\section{Results}

The diabetic group consisted of (1.) patients with type I DM constitutes $(\vee \%)$ and $(I r)$ patients with type $r D M$ constitutes ( 9 \%\%).

The duration of type 1 DM ranged from $0.0^{\circ} \cdot{ }^{\circ}$ years. The duration of type $r$ DM ranged from $7 . r \pm 7 . r$ years.

Both groups were classified into three subgroups according to the duration of DM. In ( $V Y)$ diabetics $(0 \leqslant \%)$ the duration was less than 0 years group while in $r q(r \wedge \%)$ the duration was between $\left.0_{-}\right)$. years groups and in $r \tau$ ( $11 \%)$ the duration was more than 1. years. The mean BMI \pm SD of non diabetic group were rฯ.or $\pm \leqslant . \cdot \varepsilon$ vs. Y . $r \vee \pm \Sigma . \wedge$. for diabetic group. From the $11 \overline{0}$ control subjects $(n=1 r)(11 \%)$ were hypertensive and from $1 \leqslant r$ diabetic patients $(n=7 \leqslant)(\leqslant 0 \%)$.were hypertensive. The mean systolic blood pressure in non diabetic and diabetic group were $1 r \cdot \pm 1 \cdot$ and $r \cdot+\underline{r} \cdot \mathrm{mmHg}$ respectively. The mean diastolic blood pressure in non diabetic and diabetic group were $\Lambda \cdot \pm 1 \cdot, \Lambda^{\circ} \pm 1 \cdot \mathrm{mmHg}$ respectively. 
Four subjects $(\ulcorner .0 \%)$ had history of IHD in non diabetic group while r $\vee(19 \%)$ diabetic patients had a history of IHD, all of these twenty seven diabetics were of typer DM..Patients with IHD in both groups had heart disease for more than one year and they were on regular therapy for their conditions .The results of different biochemical parameters were presented in table (').

Effect of hypertension on the biochemical parameters within the diabetic group: No statistical significant difference was noticed in $\mathrm{FPG}, \mathrm{HbA} / \mathrm{C}$, HDL-C, uric acid between hypertensive and non hypertensive diabetics, while a significant difference in T-cholesterol and LDL-C measurements between hypertensive and non hypertensive diabetics Table( $r$ ).

\section{Effect of hypertension on biochemical parameters in the studied groups:}

A highly significant differences in the T-Cholesterol, Triglycerides, LDL-C and HDL-C were detected; the difference in uric acid was not significant.

About one fifth of diabetic patient $(19 . . \%)$ had history of IHD diagnosed by physician and they were on treatment, in comparison to only $r .0 \%$ of non diabetic group, a patient with diabetes has almost tow folds risk for the development of IHD than the non diabetic subject (Odd ratio $=\$ 9.90$, $\mathrm{P}=\cdot, \cdots 1)$.

Comparison between diabetic with IHD and diabetic without IHD:

Within cases study for diabetic patient, with IHD and those without IHD showed higher $\mathrm{HbA}{ }^{\prime} \mathrm{C}$, worse Lipid profile, lower uric acid and higher blood pressure in diabetic with IHD than those without, but the difference is statistically not significant. Significant difference in duration of DM $(\mathrm{P}<\cdot . \cdot) \cdot$ was detected, the duration was longer in those with IHD than those without. Table $\left({ }^{\circ}\right)$.

Table (1): Biochemical parameters of the studied groups.

\begin{tabular}{|c|c|c|c|c|c|c|c|}
\hline \multirow{2}{*}{ Biochemical parameters } & \multicolumn{3}{|c|}{ Non diabetic group $n=110$} & \multicolumn{3}{|c|}{ Diabetic group $n=1 \leqslant r$} & \multirow{2}{*}{ P-value } \\
\hline & Mean+SD & Median & Range & Mean+SD & Median & Range & \\
\hline $\mathrm{FPG}(\mathrm{mmol} / \mathrm{L})$ & $\varepsilon . Y^{q} \pm \cdot .7 \varepsilon$ & 5.19 & $r_{.} \cdot T_{-1.0}$ & q.VI+r.ro & $9 . \leqslant r$ & $r .0 \Lambda_{-} 19 . \wedge$. & $\because \cdots 1$ \\
\hline $\mathrm{HbA} / \mathrm{c} \%$ & $\varepsilon . r Y \pm \cdot V \varepsilon$ & $\varepsilon . \mu$ & $r . \cdot 0 . \wedge$ & $0 . v \pm 1 . \leqslant V$ & $\varepsilon . \wedge$. & $r .0 \cdot-1 \cdot . \wedge$. & $\because \cdots 1$ \\
\hline T-cholesterol $(\mathrm{mmol} / \mathrm{L})$ & $\varepsilon . \varepsilon \cdot \pm \cdot .91$ & $\varepsilon . \varepsilon \wedge$ & r.r. & $0.7 r+1.00$ & 0.57 & $r . r 7-1 \cdot r u$ & $\because \cdots 1$ \\
\hline Triglycerides (mmol/L) & $1 . P \pm \cdot . Y r$ & .911 & $\left.\cdot . \leqslant \Lambda_{-}\right) .90$ & T.TY+1.Tr & T.YT & $\cdot .07-9.9$ & $\because \cdots 1$ \\
\hline $\mathrm{HDL}-\mathrm{C}(\mathrm{mmol} / \mathrm{L})$ & $1 . r 1 \pm \cdot .11$ & 1.41 & $\because V T-1 . Y \Lambda$ & $1 . V \pm \cdot r r$ & $1 . \cdot 1$ & $\because \leqslant 1-1.1$ & $\because \cdots 1$ \\
\hline LDL-C (mmol/L) & $r .0 \leqslant \pm .90$ & $r . \varepsilon r$ & $1.19-5.77$ & $r .9 \cdot+r .1 \mathrm{~V}$ & r.0 & $1 . V-r \cdot . \Lambda \Lambda$ & $\because \cdots 1$ \\
\hline S.Uric acid $(\mu \mathrm{mol} / \mathrm{L})$ & $r \cdot r . \varepsilon+7 \Lambda . \varepsilon$ & MIT & $100 \_\leqslant \leqslant \leqslant$ & M10+VI.z & rqs & $1 \leq \varepsilon-1 \cdot \leq \varepsilon$ & $\cdot .179$ \\
\hline
\end{tabular}

All values are expressed as mean $\pm \mathrm{SD}, \mathrm{FPG}=$ fasting plasma glucose, $\mathrm{HDL}-\mathrm{C}=$ high density lipoprotein cholesterol, LDL-C=low density lipoprotein cholesterol

Table $(r)$ : Effect of hypertension on biochemical parameters in the studied groups.

\begin{tabular}{|c|c|c|c|}
\hline "Biochemical parameters & $\begin{array}{c}\text { Hypertensive non diabetics } \mathrm{n}=1 \mathrm{~T} \\
\text { mean+SD }\end{array}$ & $\begin{array}{c}\text { Hypertensive diabetics } \\
\mathrm{n}=\tau \varepsilon \text { mean+SD }\end{array}$ & P-value \\
\hline T-cholesterol (mmol/L) & $0 . r \cdot \pm \cdot .09$ & $7.1 \mathrm{Y} \pm 1.0 \mathrm{~V}$ & $\because \cdots 1$ \\
\hline Triglycerides (mmol/L) & $1.20 \pm \cdot . Y V$ & $r . V \varepsilon \pm \cdot . V V$ & $\because \cdots 1$ \\
\hline LDL-C (mmol/L) & $r .0 Y+\cdot .74$ & E.rr+1.VT & $\because \cdots \wedge$ \\
\hline HDL-C (mmol/L) & $1 . Y \wedge \pm \cdot . Y 7$ & $1 . \varepsilon \pm \cdot \cdot{ }^{m}$ & $\because \cdots$ \\
\hline Uric acid $(\mu \mathrm{mol} / \mathrm{L})$ & $r r \cdot .7 V+01.7$ & $r 19 . r+1 M 1 . \Sigma$ & $\cdot .7 \cdot r$ \\
\hline
\end{tabular}

All values are expressed as mean $\pm \mathrm{SD}, \mathrm{FPG}=$ fasting plasma glucose, $\mathrm{HDL}-\mathrm{C}=$ high density lipoprotein cholesterol, LDL-C=low density lipoprotein cholesterol 
Table ( $($ ): Effect of hypertension on biochemical parameters, within diabetic group.

\begin{tabular}{|c|c|c|c|}
\hline Biochemical parameters & $\begin{array}{c}\text { Non hypertensive diabetics } \\
n=\vee \wedge \text { mean }+ \text { SD }\end{array}$ & $\begin{array}{c}\text { Hypertensive diabetics } n=7 \varepsilon \\
\text { mean+SD }\end{array}$ & P-value \\
\hline FPG $(\mathrm{mmol} / \mathrm{L})$ & $1 \cdot .9+r .0 r$ & $9 . r \leqslant+r .99$ & .1199 \\
\hline $\mathrm{HbA})^{\prime} \mathrm{C} \%$ & $0.10+1.01$ & $\varepsilon .10+1 . \varepsilon r$ & $.1 \leqslant$ \\
\hline T-cholesterol (mmol/L) & $0 . r \Lambda+1 . \varepsilon \varepsilon$ & $7.1 Y \pm 1.0 \mathrm{~V}$ & $\because .41$ \\
\hline Triglycerides $(\mathrm{mmol} / \mathrm{L})$ & $1 . r \pm+1.77$ & $Y . V \varepsilon \pm \cdot . V V$ & $\because \cdot \wedge \wedge$ \\
\hline LDL-C (mmol/L) & $1.00+\cdot .77$ & $\varepsilon . r+1 . V \tau$ & 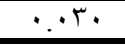 \\
\hline $\mathrm{HDL}-\mathrm{C}(\mathrm{mmol} / \mathrm{L})$ & $1.1 r+\cdot . r \mu$ & $1 . \cdot \varepsilon \pm \cdot . r 1$ & $\because 7 \wedge$ \\
\hline Uric acid $\mu \mathrm{mol} / \mathrm{L}$ & $r \cdot \varepsilon . \wedge V \pm 1 \cdot r$ & $r 19 . r+1 r 1 . \varepsilon$ & $\because$ rOA \\
\hline
\end{tabular}

All values are expressed as mean $\pm \mathrm{SD}, \mathrm{FPG}=$ fasting plasma glucose, $\mathrm{HDL}-\mathrm{C}=$ high density lipoprotein cholesterol, LDL-C=low density lipoprotein cholesterol

Table ( $\varepsilon)$ : Association of diabetes and IHD .

\begin{tabular}{|c|c|c|c|}
\hline \multirow{2}{*}{} & \multicolumn{3}{|c|}{ Ischemic heart disease } \\
\cline { 2 - 4 } & Present & absent & Total \\
\hline Diabetic group & $r V$ & 110 & $1 \leqslant r$ \\
\hline Non Diabetic group & $\varepsilon$ & 111 & 110 \\
\hline Total & $r$ & $r r r$ & rov \\
\hline
\end{tabular}

Odd ratio $=£ 9.90$

$\mathrm{P}=\cdot \cdots+1$

Table ( $\left.{ }^{\ominus}\right)$ : Comparison between diabetic patients with IHD and without IHD.

\begin{tabular}{|c|c|c|c|}
\hline Biochemical parameters & $\begin{array}{l}\text { Diabetic without IHD } n=110 \\
\text { mean }+ \text { SD }\end{array}$ & $\begin{array}{l}\text { Diabetic with IHD } n=r v \\
\text { mean }+ \text { SD }\end{array}$ & P-value \\
\hline FPG (mmol/L) & 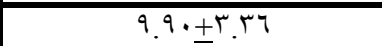 & $\wedge . q \cdot \pm r \cdot Y V$ & .171 \\
\hline $\mathrm{HbA} / \mathrm{C} \%$ & $0.9 \pm 1.01$ & $0.1 \cdot \pm 1 . r \cdot$ & $\cdot .91 \mathrm{r}$ \\
\hline T-cholesterol (mmol/L) & $0.0 \cdot \pm 1 . \leqslant 7$ & $7.11+1 . \vee \wedge$ & $\because \times 7$ \\
\hline Triglycerides $(\mathrm{mmol} / \mathrm{L})$ & $r .70 \pm 1.01$ & $r .9 \wedge \pm 1 . \wedge r$ & $.70 \mathrm{r}$ \\
\hline LDL-C (mmol/L) & $r . \wedge \cdot \pm r . Y \varepsilon$ & $\{. r \pm 1 . \wedge 1$ &. .171 \\
\hline HDL-C (mmol/L) & $1 . A_{ \pm} \cdot . r \mu$ & $1.0 \pm \cdot .11$ & $\cdot .7 \cdot 1$ \\
\hline Uric acid $(\mu \mathrm{mol} / \mathrm{L})$ & $r \wedge .7 \pm 1 r r$ & $r \cdot 1 . \wedge \pm 1 \cdot r .7$ & $\cdot r \leqslant \Lambda$ \\
\hline Duration of DM (years) & $0 . r V \pm 0 . r$. & $1 \cdot Y Y_{ \pm} \Lambda . V T$ & $\because 1 \cdot$ \\
\hline BMI & rq.ro+z.qr & $r q . \Sigma V \pm \varepsilon . r q$ & $\cdot .9 \cdots$ \\
\hline Diastolic (mmHg) & $\wedge \varepsilon, \cdots \pm 11, \cdots$ & Av....$\cdots$ & $\because 11 Y$ \\
\hline Systolic Bp (mmHg) & $T r \cdot \cdots \pm r \cdot . \cdots$ & $1 \varepsilon \cdot \cdots \pm+\cdot \cdots$ & $\because 7 \varepsilon$ \\
\hline
\end{tabular}

All values are expressed as mean \pm SD

$\mathrm{FPG}=$ fasting plasma glucose

HDL-C $=$ high density lipoprotein cholesterol

LDLC $=$ low density lipoprotein cholesterol

BMI:Body mass index. 


\section{Discussion}

The third report of the Expert panel on Detection, Evaluation and treatment of high blood cholesterol in adult (ATPIII) made diabetes a coronary heart disease equivalent, thereby elevating it to the highest risk category ${ }^{(' \vee}$.

Diabetologists know well that insulin and hypoglycemic drugs are only a small part of the weaponry against this ancient and dangerous disease. Patient education, special clinics and regular laboratory checking are all important strategies in the fight to improve glycemic control and to reduce the complications ('^). The rising world wide rates of DM heightened the need to maintain adequate metabolic control and control for other cardiovascular risk factors, such as lipid profile disturbances and high blood pressure ${ }^{(19)}$.

Patients with diabetes have a spectrum of lipid abnormalities that may be associated with increased risk of developing IHD. This increase in risk may be due in part to qualitative difference in the lipoprotein fraction or presence of other atherogenic changes such as small dense LDL and oxidized LDL $^{(r \cdot)}$. One of the most common type of hyperlipidemia in diabetes is modest hypertriglyceridemia (r) which is recognized now as an independent risk factor for IHD, it has atherosclerosis accelerating role by driving cholesterol into the intema of blood vessels ${ }^{(r)}$.

In this study non of type 1 diabetics have IHD,but they shared type $r$ diabetics their lipid disturbances.

The pattern of dyslipidemia within diabetics in the present study was combined hyperlipidemia (hypercholesterolemia and hypertriglyceridemia with low HDL-C), which is consistent to that reported by different local studies in Mosul ${ }^{(\Gamma, Y \varepsilon)}$.

However It differs from that stated by Arora et al, ${ }^{\left({ }^{\circ}\right)}$ in that no significant differences have been found in total cholesterol and LDL-C measurements between diabetic and non diabetic individuals. Lipid pattern in patients with type $r$ DM, insulin resistance and relative insulin deficiency are associated with hypertriglycerdemia ,low HDL-C and occasionally high LDL-C ${ }^{(\Upsilon)}$.

The treatment of hypertension in diabetics might decrease the risk of stroke, however, the reduction in IHD is controversial. The reason for this differential effect is keenly debated. It Could be attributed to the fact that antihypertensive treatment with thiazide diuretic and B-blockers has adverse cardiovascular effects through induced lipid changes and glucose intolerance $^{\left({ }^{Y}\right)}$.

In the present study total cholesterol and other lipids are worse in hypertensive diabetics than non diabetics and this may be attributed to diabetes itself., this finding is consistent to that stated by El-Kebbi, et al, ${ }^{(\uparrow \wedge)}$. The total cholesterol and LDL-C were significantly higher in hypertensive compared to non hypertensive diabetics patients which is in line to that reported by Shaker, ${ }^{\left({ }^{q}\right)}$ and Kanaoun, ${ }^{(r \cdot)}$. This may focus the attention to more recent interest about the association between DM, hypertension and hypercholesterolemia namely the metabolic syndrome $^{\left({ }^{\prime}\right)}$. It seems that those hypertensive dyslipidemic diabetic patients are the major risk group within diabetics themselves. Recent recommendation for the treatment of dyslipidemia and hypertension in diabetic patients suggest that such patients should be treated as aggressively as those with preexisting IHD, a recommendation that is reasonable if DM confers the same level of risk as IHD. ${ }^{(r)}$.

One of the unresolved questions is whether insulin resistance and/or hyperinsulinemia rather than hyperglycemia is the main risk factor for IHD in DM as it is associated with abnormal lipid, hypertension, prothrombotic phenomena on which are contributing to atherosclerosis and $\operatorname{IHD}^{(r)}$. The 
importance of glycemic control on the development of macro vascular complication is an area of controversy. The present study showed no significant difference in regard to glycemic control between diabetics with IHD to diabetics without IHD. On the other hand significant difference is found concerning the duration of diabetes. Those with IHD had longer duration of DM. It is probably that the longer the diabetic exposed to hyperglycemia the more accumulative effect of glycated end products and the more exposure to disturbed lipid in both quantity and quality. Hyperlipidemia is a predictor of IHD, with independent and graded positive association between cholesterol and IHD ${ }^{()^{)}}$. One local study in Mosul by Al-Naemy ${ }^{\left({ }^{(\varepsilon)}\right)}$ showed that no significant difference in both triglycerides and serum uric acid between those with IHD to those without.

In conclusion, diabetic patients have substantially increased risk for ischemic heart disease, including biochemical \& non biochemical risk.. Diabetic patients have different patterns of dyslipidemia,

It is recommended to consider lipidlowering agents in these high risk patients, with a full understanding of their action and side effects. Hypertensive diabetics seem to be the major risky group within the diabetic themselves. Tight control of blood pressure goes along with optimal glycemic control are mandatory tools.

\section{References}

1.FrierBM,Truswell AS, Shepherd J,de Looy A, Jung R.Diabetes mellitus and nutritional and metabolic disorders. In: Haslett C,Chilvers ER,Hunter JA,Boon NA(Eds). Davidson's principles and practice of medicine, $1 \wedge^{\text {th }}$ ed,Churchill living-stone, Edin-burgh, U.K; 1999; pp $\leqslant \vee r_{-} \leqslant \wedge r$

r.McCulloch DK. Definition and classification of diabetes mellitus. up to date.CD. www.Up todate.com. $(9 \cdots)$ r.. r: rrrv_sVAᄉ.
r.Donnelly R, Davis KR. Type r diabetes and atherosclerosis. Diabetes, obesity and metabolism. $r \ldots$; (suppl): or __or.

$\{$.Anonymeus. Diabetes and coronary heart disease.... .Website: http://www.chebucto.ns.ca/health.

-.CDC Diabetes, Cost. Effectiveness Group. Cost-effectiveness of intensive glycemic control, Intensified hypertension control and serum cholesterol level reduction for typer diabetes JAMA r... F; rAv: ros rroo1.

7.McKinght JA .Managing blood pressure in type $r$ diabetes mellitus. Practical diabetes Int. $r \ldots$; I $\vee(r)$ : ¿9_or.

$\checkmark$.Allison. DB ,Sanders SE .Obesity in North America. The medical clinics of North America. $r \cdots ; \wedge \leqslant(r): \leqslant \leqslant 0_{-} \leqslant T \vee$.

^.Leermakers EA, Dunn AL, Blair SN. Exercise management of obesity. Medical clinics of North American. $r \ldots ; \wedge \leq(r)$ : $\leqslant 1 q_{-} \leqslant$ro.

१.Teitz NW, Burtis CA, Ashwood ER. Text book of clinical chemistry $r^{\text {nd }}$ ed., Saunders, Philadelphia, USA; $199 \leqslant: \vee \cdots, 90 \Lambda_{-} 970$.

1..Moore JC, Bown E, Outlaw MC, Jelfs R, Holman RR, Jurner RC. Comparison of five different methods, including measurement on capillary blood samples. Ann. Clin. Bio-

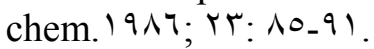

1).Artiss JD, Entwistle wm. The application of a sensitive Uricaseperoxides coupled reaction to a centrifugal fast analyzer for the determination of uric acid .Clin. Chem. Acts. 1911; 117: r.1 -r.9.

I Y.Virdi NK, Worthington DJ. Cholesterol and triglyceride stability in whole blood Ann. Clin. Biochem. 1919; r7: 19V_191.

$1 \%$.Lopez - Virella MF, Stone P, Ellis S, Cowell JA. Cholesterol determination in HDL separated by three different methods. Clin.Chem. I $9 v \mathrm{~V}$; rr (s): ANY_AN .

1 ร.Hoefner DM, Hodel SD, Obrin JF, Branum EL, Sun D, Meissner I et al. 
Development of a rapid quantitative method for LDL, sub fractionation with use the quantimetrix lipoprint LDL system. Clin. Chem. $r .$. ।; $\leqslant \vee(r)$ : YTI_rV纟.

10.Bourke GJ, McGilvray J .Interpretation and uses of medical statistics, $r^{\text {nd }}$ ed. Blackwell, oxford, London, UK, I $9 \vee \wedge$; PP $\leqslant$ ᄉ_o .

17. Armitage P. Statistical methods in medical research, $\varepsilon^{\text {th }}$ printing. Blackwell, Oxford, London, UK; |9V $\leqslant: P 1 \cdot 7$.

IV.Executive summery of the third report of the national cholesterol education program (NECP) Expect panel on detection, evaluation and treatment of high blood cholesterol in adults (Adult treatment III) ATPIII

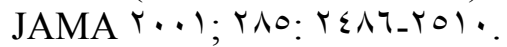

'^.Gill GV. Financing diabetes health care.. practical diabetes Digest. 199.; l( $(\mathrm{T}):$..

19.Bilo. HJ, Gans. RO . Hypertensive patients and diabetes: A high risk population. J. Cardiovascular Pharmacol. 199^; rY(Y): 0 1_^ (Abstract)

$r \cdot$ McCulloch DK, Rosenson RS. Management of dyslipidemia in diabetic patient. up to date $\mathrm{CD}$. www.up to date .com $\wedge \ldots . r \ldots r$ : (99人-7T\&).

$r$.Betteridage DJ. Diabetic dyslipidemia. Diabetes, obesity and Metabolism. r..r; r( ( ): or L_orr.

$r$ r.Rader DJ, Rosas S. Management of selected lipid abnormalities. Medical clinics. of North America. r...; $\wedge \varepsilon(1): \leqslant r-7 r$

rr.Abdul-Razzak NA, Mula-Abid WS Glycemic indices and other biochemical parameters in Iraqi diabetics. J. Fac .Med. Baghdad. 199v; \&. ( $(Y)$ : IVq_ INV.

$r$ \&.Al-Hamdani RY. Pattern of dyslipidemia in diabetic patients I. J. Basic Med. Sc. Y..r.r (r): $\left.1 \cdot V_{-}\right) 1 \cdot$.

ro.Arora S, Caballero AE, Sauaf R Micro vascular and macro vascular reactivity is reduced in subjects at risk for type $r$ diabetes. Diabetes. $r \ldots r$;

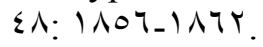

rฯ.Watts Gf. Coronary disease, dyslipidemia and clinical trials in type $r$ diabetes mellitus. Practical diabetes Int. $r \cdots ; Y Y(r): 0 . .09$.

YV.Leese GP and Vora JP). New aspects of the treatment of hypertension in diabetes. Practical diabetes int. 1991;10 (r): Yrr_rs...

r^.El-Kebbi IM, Ziemer DC, Cook CB, Miller CD, Gllina DL, Philips LS Co morbidity and glycemic control in patients with type $r$ diabetes. Arch.

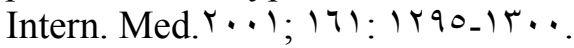

$r 9$.Shaker M. Pattern of dyslipidemia in diabetic patients.Msc thesis in Biochemistry, college of Medicine ,University of Basrah, 1991..

$r \cdot$.Kanoun F, Ben Amor Z, Zouari B, Ben Khalifa $F$. Insulin therapy may increase blood pressure levels in type diabetes mellitus. Diabetes mellitus. ${ }^{\top} \cdots r$; $r \vee(\uparrow): ~ 790-\vee \cdots$.

$)$.Ford ES, Giles WH, Dietz WH. Prevalence of the metabolic syndrome among US Adults. J.A.M.A.Y..r; YNV: ror_roq.

$r$ r.Haffner SM .Coronary heart disease in patients with diabetes. New. Eng. J .Med. $r \cdot r ; r \leqslant r(1 \leq): 1 \cdot \leq \cdot-1 \cdot \leq r$.

$r$.Bell DSH. Advances in diabetes for the Millennium: The heart and diabetes. ${ }^{\prime} \cdot \leqslant$; ; Internet communication

$r \varepsilon$.Al-Neamy AA. Autonomic neuropathy and silent myocardial ischemia in type $r$ diabetic patients. A thesis for fellowship of the Iraqi commission for Medical Speciali-zation. University of Mosul,,$\ldots$..'. 
